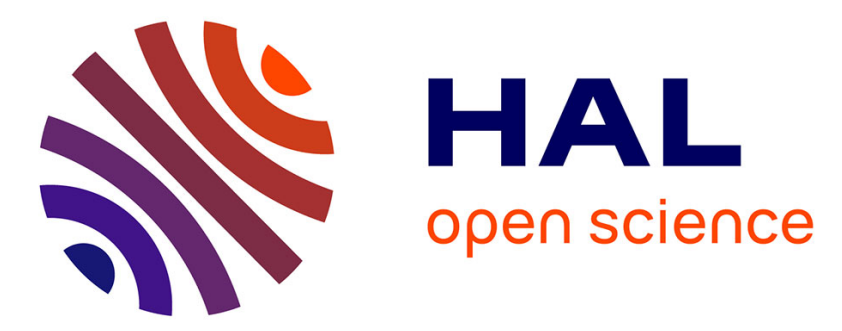

\title{
Personality change associated with chronic diseases: pooled analysis of four prospective cohort studies.
}

\author{
Markus Jokela, Christian Hakulinen, Archana Singh-Manoux, Mika Kivimäki
}

\section{To cite this version:}

Markus Jokela, Christian Hakulinen, Archana Singh-Manoux, Mika Kivimäki. Personality change associated with chronic diseases: pooled analysis of four prospective cohort studies.. Psychological Medicine, 2014, 44 (12), pp.2629-40. 10.1017/S0033291714000257 . inserm-01151631

\section{HAL Id: inserm-01151631 https://www.hal.inserm.fr/inserm-01151631}

Submitted on 13 May 2015

HAL is a multi-disciplinary open access archive for the deposit and dissemination of scientific research documents, whether they are published or not. The documents may come from teaching and research institutions in France or abroad, or from public or private research centers.
L'archive ouverte pluridisciplinaire HAL, est destinée au dépôt et à la diffusion de documents scientifiques de niveau recherche, publiés ou non, émanant des établissements d'enseignement et de recherche français ou étrangers, des laboratoires publics ou privés. 


\section{Personality Change associated with Chronic Diseases: Pooled Analysis of Four}

\section{Prospective Cohort Studies}

Markus Jokela, ${ }^{1,2^{*}}$ Christian Hakulinen, ${ }^{1}$ Archana Singh-Manoux, ${ }^{3,4}$ Mika Kivimäki ${ }^{3}$

${ }^{1}$ Institute of Behavioural Sciences, University of Helsinki, Finland

${ }^{2}$ Department of Psychology, University of Cambridge, UK

${ }^{3}$ Research Department of Epidemiology and Public Health, University College London, UK

${ }^{4}$ Inserm U1018, Centre for Research in Epidemiology and Population Health, Villejuif, France

Funding sources: This research was supported by the Academy of Finland (grant numbers: 124322, 124271 and 132944); Medical Research Council (MRC K013351); the US National Institutes of Health (R01HL036310; R01AG034454); and the Finnish Work Environment Fund. Christian Hakulinen was financially supported by the Research Foundation of the University of Helsinki and the Finnish Cultural Foundation, Archana Singh-Manoux was supported by the National Institute on Aging, NIH (R01AG013196; R01AG034454). Mika Kivimäki is an Economic and Social Research Council Professor. None of the authors have any competing interests.

Corresponding author: Markus Jokela, Institute of Behavioural Sciences,

Siltavuorenpenger 1A, P.O. Box 9, 00014 University of Helsinki, Finland. Telephone: +3589-19129483, E-mail: markus.jokela@helsinki.fi

Manuscript statistics: 225 words in abstract, 4891 words in text, 2 Tables, 2 Figures + Webonly supplementary material (16 pages with 13 figures) 


\begin{abstract}
Background. Common chronic conditions, such as heart disease and cancer, are associated with increased psychological distress, functional limitations, and shortened life expectancy, but whether these diseases alter aspects of personality remains unclear.

Methods. To examine whether the onset of heart disease, stroke, diabetes, cancer, hypertension, arthritis, and respiratory disease is associated with subsequent changes in personality traits of the five-factor model, we pooled data from the Health and Retirement Study, the Midlife in the United States Survey, and the graduate and sibling samples of the Wisconsin Longitudinal Study for an individual-participant meta-analysis (total $n=17,493$; mean age at baseline 55.8 years).
\end{abstract}

Results. After adjustment for age, we observed consistent decreases in extraversion $(-0.25 \mathrm{~T}$ scores per 1 disease; $95 \%$ confidence interval=- $0.40,-0.10)$, emotional stability $(-0.40 ;-0.61$, $-0.19)$, conscientiousness $(-0.44 ;-0.57,-0.30)$, and openness to experience $(-0.25 ;-0.37,-$ $0.13)$ but not in agreeableness $(-0.05 ;-0.19,0.08)$ after the onset of chronic diseases. The onset of each additional chronic disease accelerated the average age-related personality change by 2.5 years in decreasing extraversion, 5.5 years in decreasing conscientiousness, and 1.6 years in decreasing openness to experience, and attenuated the increasing levels of emotional stability by 1.9 years. Comorbid conditions were associated with larger changes than single diseases, suggesting a dose-response association between morbidity and personality change.

Conclusions. These results support the hypothesis that chronic diseases influence personality development in adulthood.

Keywords: Personality development; Morbidity; Chronic disease; Longitudinal; Metaanalysis 
Chronic diseases account for a substantial proportion of disability and premature mortality in the developed countries as well as globally (Wang et al. 2012). Adaptation to these illnesses constitutes an important psychosocial transition in the human life-course, as chronic conditions are likely to affect the daily activities of most individuals at some point in their lives (Katz \& Yelin 1995; Affleck et al. 2001; Crimmins 2004). There is an extensive research on the negative emotional reactions associated with chronic diseases (Stanton et al. 2007; de Ridder et al. 2008) as well as on sociodemographic factors and psychological coping styles that may modify these reactions (Davydov et al. 2010; Evers et al. 2011). However, the broader implications of chronic diseases to personality development have not been studied in detail. These personality changes are not well captured by narrow concepts of psychological distress. Personality change related to chronic diseases is also relevant for psychiatry and clinical psychology, as enduring personality change due to the physiological effects of a medical condition is one of the diagnostic categories of personality disorders. We investigated whether common chronic diseases affect the long-term development of personality traits assessed with the more comprehensive five-factor model of personality.

\section{Adaptation to Chronic Diseases}

The onset of a chronic disease involves several health-related physiological, psychological, and social changes (Penley et al. 2002; Sharpe \& Curran 2006). Some chronic diseases, such as stroke and many cancers present special challenges for those who survive these diseases, as the risk of recurrence is often high (Go et al. 2013). Other diseases, such as arthritis, respiratory diseases, and heart disease, often cause considerable disability and functional limitations, and increase mortality risk in the long term (McCathie et al. 2002; Strong et al.2005; Evers et al. 2011). Medication and other treatments alleviate symptoms of most common chronic diseases to some extent, but a substantial proportion of individuals 
with chronic diseases do not follow their prescribed treatments (Osterberg \& Blaschke 2005), which weakens the effectiveness of available medical care.

Despite the substantial distress and functional limitations associated with chronic diseases (Prince et al. 2007; Stanton et al. 2007; de Ridder et al. 2008), many people are able to adjust to physical illnesses over time, so not all mental health problems caused by chronic illnesses are permanent (Wu 2001; Powdthavee 2009). Indeed, many studies have shown that people tend to regain their earlier levels of wellbeing after negative life events (Clark et al. 2008; Powdthavee 2009; Davydov et al. 2010), although this is not always the case (Headey 2010). Moreover, surviving and learning to live with a chronic disease may prompt personal growth in some individuals (Sheikh 2004), suggesting that negative and positive experiences may coexist in adaptation to chronic diseases (Barskova \& Oesterreich 2009; Bostock et al. 2009). It is therefore difficult to extrapolate the long-term psychological impact of chronic diseases from the short-term distress reactions to disease onset.

Most of the earlier studies have investigated adaptation with measures of psychological distress, quality of life, physical pain, limitations in daily activities, or disease progression, but not with complete personality inventories. In clinical practice, changes in personality are often reported especially in stroke survivors (Remer-Osborn 1998). In one retrospective study, individuals recovering from stroke $(n=35)$ were rated by their carers to be more bored, frustrated, dissatisfied, unhappy, irritable, worried, and unreasonable after compared to before stroke (Stone et al. 2004). Another retrospective study based on informant-rated personality before and after stroke $(n=70)$ found increased levels of depression and communication difficulties but no change in indifferent, inappropriate or manic behavior (Nelson et al. 1993).

Disease-related changes in personality have also been studied in neurodegenerative diseases, such as Alzheimer's disease (Robins Wahlin \& Byrne 2010), Parkinson's disease 
(Mendelsohn et al. 1995), dementia (Gao et al. 2000), and in cases of brain injury (Obonsawin et al. 2007). Like stroke, these conditions directly affect brain functioning, and they can also be expected to pose major challenges of adjustment and recovery. The majority of these studies have assessed personality change by comparing current and retrospective assessment of personality characteristics evaluated by family members (Robins Wahlin \& Byrne 2010). Considerable decreases (between 1 and 3 standard deviations) in levels of conscientiousness (e.g., forgetfulness, difficulties in persisting with tasks at hand), emotional stability (e.g., symptoms of depression), and agreeableness (e.g., interersonal conflicts) in individuals with brain-related diseases or injuries have been reported (Robins Wahlin \& Byrne 2010). However, it is difficult to evaluate to what extent such changes represent the outcome of brain pathology or patterns of personality change related to chronic diseases in general.

Besides the above studies on stroke and neurogenerative diseases, we were unable to find any longitudinal studies comparing people's personality before and after the onset of common chronic diseases, such as diabetes and heart disease. Thus, research on the implications of common chronic diseases for long-term personality development appears to be scarce. In particular, there is a lack of prospective longitudinal data with standardized personality measures.

\section{The Present Study}

We carried out an individual-participant meta-analysis with more than 17,000 participants from four cohort studies to examine whether the onset of a chronic disease heart disease, stroke, diabetes, cancer, respiratory disease, arthritis, and hypertension among individuals initially free of the disease is associated with subsequent changes in personality traits, measured using the five-factor model (extraversion, emotional stability, 
agreeableness, conscientiousness, and openness to experience). More specifically, we ask whether chronic conditions accelerate or reverse the average age-related personality trajectories, or whether personality remains largely unchanged despite the appearance of physical morbidities. Prospective longitudinal data with repeated measurements of personality from 4 cohorts provided a methodologically robust setting to examine these associations.

Based on previous studies of chronic diseases and quality of life (Stanton et al.2007; de Ridder et al. 2008) we hypothesized that the onset of a disease would be associated with a decrease in emotional stability due to psychological distress, disability, and limitations to daily activities caused by the disease. We also hypothesized a decrease in extraversion, because several chronic diseases cause fatigue and loss of energy (de Ridder et al.2008) that potentially decrease features of extraversion, such as positive mood, activity, and approachmotivated behavior. Furthermore, some studies have suggested that suffering from physical illnesses increases irritability and interpersonal conflicts, perhaps because of a lowered level of patience or concern for other people's troubles (Stanton et al. 2007). We therefore hypothesize that such changes might decrease agreeableness. Decreases in conscientiousness and openness have been reported in chronic diseases affecting the brain (Robins Wahlin \& Byrne 2010), but we did not have sufficient a priori evidence to evaluate whether a similar change would be expected in relation to other chronic diseases. Finally, we hypothesize that the personality change associated with stroke would be more marked than those for other chronic diseases studied, that is, heart disease, diabetes, hypertension, cancer, respiratory disease, and arthritis, because stroke affects brain functioningone . Besides this diseasespecific hypothesis, we had no other hypotheses regarding differences between the 7 chronic diseases included in the present study. 


\section{Methods and Materials}

The data for the individual-participant meta-analysis were selected by searching the collections of the Inter-University Consortium for Political and Social Research (ICPSR; http://www.icpsr.umich.edu/icpsrweb/ICPSR/) to identify large-scale cohort studies with repeated measurements of personality and chronic diseases. We included all studies with repeated measurements of personality assessed using the five-factor model; information on specific chronic health conditions; and large sample size $(n>1000)$. The following studies met these inclusion criteria: the Health and Retirement Study; the Midlife in the United States; and the Wisconsin Longitudinal Study graduate and sibling samples.

Full details of the cohorts and measures are provided in the Online Supplementary Material. Briefly, the Health and Retirement Study (HRS) is a nationally representative longitudinal study that started in 1992 and altogether includes more than 30,000 individuals representing the U.S. population older than 50 years (Juster \& Suzman 1995). Midlife in the United States (MIDUS) is based on a nationally representative random-digit-dial sample $(n=7,108)$ of non-institutionalized, English-speaking adults, aged 25 to 74 years in 1995-1996 (Brim et al. 2007). The Wisconsin Longitudinal Study (WLS) is a study of 10,317 participants who were born between 1937 and 1940 and who graduated from Wisconsin high schools in 1957 (Wollmering 2007). In addition to the main sample of the 1957 high school graduates, the WLS has also collected data on a selected sibling of a sample of the graduates. The data collection in adulthood has been very similar although not entirely identical for the siblings as for the graduates. Although the graduate (WLSG) and sibling (WLSS) samples are collected in the same study, we treated them separately because the sibling sample is more heterogeneous in terms of age compared to the graduate sample.

\section{Measures}


In all studies, personality was assessed using one of the standardized questionnaire instruments based on the five-factor model of personality (see details of the measures in Online Supplementary Material). The instruments measure five higher-order personality traits that sum up individual variation in several more specific personality dispositions. Extraversion reflects characteristics such as social assertiveness, sociability, and sensitivity to positive emotions; individuals with high emotional stability (or low neuroticism) are not easily distressed or sensitive to negative emotions, they are resilient in stressful situations, and seldom experience feelings of anxiety, sadness or depression; agreeableness measures cooperativeness, altruism, and trust toward other people; conscientiousness is expressed as self-control, orderliness, and adherence to social norms; and openness to experience correlates with curiosity, broad-ranging interests, and open-mindedness. For comparison, we examined corresponding changes in self-rated health and depressive symptoms (or negative mood) over the same follow-up period as the analysis of personality change. The seven common chronic conditions included in the analysis were self-reported. The participants of each study were asked to report whether they had been diagnosed of and/or treated for: heart disease, stroke, respiratory disease, diabetes, cancer, arthritis, and hypertension (see detailed questions in the online supplementary material).

\section{Statistical analysis}

Personality change was assessed by predicting personality trait score at Time 2 by chronic disease onset between Time 1 and Time 2 among individuals without the disease of interest at Time 1, adjusting for personality trait score at Time 1, sex, age, race/ethnicity, the length of follow-up between Time 1 and Time 2 (in months), and the number of other chronic diseases besides the disease of interest as the outcome variable at Time 1. A corresponding analysis was carried out for depressive symptoms and self-rated general health (instead of 
personality traits). In addition to examining each chronic disease separately, we predicted personality change by the number of chronic diseases between Time 1 and Time 2 in the total sample, adjusting for the number of chronic diseases at baseline and the other covariates listed above. This analysis enabled us to evaluate the dose-response nature of the relationship between chronic conditions and change in personality. Finally, we examined whether concurrent changes in self-rated health and depressive symptoms associated with the onset of diseases accounted for the changes in personality traits by predicting personality change with a continuously coded number of diseases between Time 1 and Time 2 (adjusted for the other covariates listed above) and then added change scores of self-rated health and depressive symptoms in the models.

To facilitate interpretation of effect sizes, all outcome variables (i.e., personality traits, depressive symptoms and self-rated general health) were first transformed into Tscores $($ Mean $=50$, standard deviation=10) before analysis using means and standard deviations at Time 1 as the metric to standardize scores at both Time 1 and Time 2 . As an additional method of quantifying the effect sizes, we estimated the average personality change in the 5 traits by pooling the estimates of study-specific personality change scores in a separate meta-analysis. We then calculated the corresponding number of years in average age-related personality trajectories associated with the disease-personality change. For the descriptive analysis of average personality change scores, we included only participants 50 years or older in order to avoid potential non-linear changes at younger ages. To take into account differences in follow-up times between studies, we assumed a linear association between years of follow-up and personality change; this assumption was based on data suggesting that non-linear patterns of personality change are relevant only on much longer follow-up periods over the life course than the 4 to 11 years included here (e.g., Roberts et al. 2006). In each study we transformed the raw change scores by dividing the scores by follow- 
up time (in years) and multiplying this by 5 to have the personality change per 5 years of age as the outcome variable in each study. This allowed us to compare the associations between chronic diseases and personality change across the samples relative to average personality change.

In all analyses we applied a 2-stage approach: models were first fitted separately within each cohort, and the results from the individual cohorts were then pooled using random-effect meta-analysis. Standard errors were calculated using robust estimator method to take into account the non-independence of individuals from the same households. All analyses were conducted using STATA version 12.1 (metan command for meta-analysis). Heterogeneity in the effect sizes was examined using $I^{2}$ estimates.

\section{Results}

Descriptive statistics of the study samples are presented in Table 1. The HRS participants were older and ethnically more heterogeneous than the other samples. Accordingly, the disease prevalence and onset was higher in the HRS than in MIDUS in which diseases were more prevalent than in the WLS samples.

First, a meta-analysis on all participants irrespective of their chronic disease status was undertaken to assess average age-related change in personality scores. These results indicated that among participants 50 years or older, extraversion decreased $(B=-0.50$ per 5-year increase in age, $\mathrm{CI}=-0.84,-0.16)$, emotional stability increased $(\mathrm{B}=1.03, \mathrm{CI}=0.76,1.31)$, agreeableness remained stable $(B=-0.04, C I=-0.35,0.26)$, conscientiousness decreased $(B=-$ $0.40, \mathrm{CI}=-0.70,-0.11)$, and openness to experience decreased $(\mathrm{B}=-0.79, \mathrm{CI}=-1.22,-0.35)$. The cohort-specific details of this meta-analysis are shown in Supplementary Figure S1.

The total number of new chronic diseases between Time 1 and Time 2 taken together showed that higher number of diseases was associated with decreasing extraversion, 
decreasing emotional stability, decreasing conscientiousness, and decreasing openness to experience over time (Figure 1; cohort-specific associations reported in Supplementary Figures S2-S6), while no significant association was observed for change in agreeableness. The associations followed a dose-response pattern, with each chronic condition strengthening the association in a linear fashion. The estimates for linear trends (i.e., personality change per 1 disease onset when the number of diseases was coded as a continuous variable) are reported in the first row of Table 2.

Comparing the estimates for average personality changes (Supplementary Figure S1; reported above) with estimates obtained from the association between disease onset and personality change (Table 2) suggested that the onset of a new disease accelerated the average age-related personality change by 2.5 years in extraversion $(=(-0.25 /-0.50) * 5=2.5)$, 5.5 years in conscientiousness, and 1.6 years in openness to experience. Given that average age changes and disease associations were in the opposite directions for emotional stability, the onset of each disease "reversed" the average development of emotional stability by 1.9 years.

To examine whether the extent of personality change associated with chronic disease onset was dependent on age, we refitted the models in Figure 1 by treating the number of chronic diseases between Time 1 and Time 2 as a continuous variable, and including an interaction term between age and number of chronic diseases between Time 1 and Time 2 (WLSG cohort was excluded from this analysis because of the restricted age range). There was no evidence for significant effect modification by age (all p-values for pooled interaction $>0.31$; details not shown). In a stratified analysis, the pooled associations were also substantially the same when the models in each cohort were fitted separately for participants $\leq 55$ years and $>55$ years of age (data not shown), suggesting no age interactions. 
The disease-specific associations are shown in Figure 2. Decreases in extraversion, emotional stability, and conscientiousness were observed after the onset of most chronic diseases examined, while change in agreeableness and openness was observed only after stroke and respiratory disease. The magnitude of personality change was relatively modest, the difference being 1-2 T-score units (equal of 0.1-0.2 standard deviations) between those with and without incident disease. The onset of stroke and respiratory disease showed the largest personality change while cancer, diabetes, and arthritis were associated with the least personality change. Cohort-specific results are reported in Figures S7-S13 in the Online Supplementary Material.

For comparison, we analyzed disease-related changes in self-rated health and depressive symptoms. As expected, self-rated general health decreased and depressive symptoms increased after the onset of chronic disease (Supplementary Figure S14). In the pooled analysis of the number of chronic diseases as the exposure and the those with no disease as the reference group $(B=0.00)$, self-rated health decreased by $-3.0(-3.8,-2.2),-6.4(-8.1,-4.6)$, $-10.0(-13.2,-6.7)$, and $-13.7(-18.4,-8.9)$ T-score units per $1,2,3$, and 4 or more chronic diseases, respectively. For depressive symptoms, the corresponding increase in symptoms associated with chronic disease onset was $0.77(0.43,1.10)$ for 1 disease, $1.12(0.74,1.51)$ for 2 diseases, $2.08(1.59,2.57)$ for 3 diseases, and $3.34(2.70,3.99)$ for 4 or more diseases. Changes in self-rated health were thus substantially larger than changes in personality traits or depressive symptoms, while changes in depressive symptoms were twice as large, on average, compared to changes in the 4 personality traits described above.

To examine whether the extent of personality change was related to the extent of change in depressive symptoms and self-rated health, we fitted the number of chronic conditions as a continuous (Table 2) rather than as categorical (Figure 1) measure, and then examined whether the associations between chronic disease onset and personality change attenuated 
after adjustment for depressive symptoms and self-rated health. As shown in Table 2, adjusting for change in depressive symptoms affected little the associations, while adjusting for change in both depressive symptoms and self-rated health attenuated the associations of chronic conditions with personality change in neuroticism, conscientiousness, and openness to experience by approximately one-third, and those with change in extraversion almost by two-thirds. Thus, deteriorating self-reported health after the onset of chronic diseases may account for some of the association between the onset of chronic diseases and personality change, especially in extraversion.

\section{Discussion}

The current individual-participant meta-analysis of four large prospective cohort studies suggests consistent changes in four of the five personality traits of the five-factor model associated with the onset of chronic diseases. The magnitude of these changes can be illustrated by considering how chronic diseases accelerate or decelerate the average rate of personality development after middle age. In a model adjusted for prevalent chronic diseases at baseline, the onset of each additional chronic disease between baseline and follow-up produced changes comparable to average decline in extraversion by 2.5 years, decline in conscientiousness by 5.5 years, and openness to experience by 1.6 years, while the increasing levels of emotional stability with age were attenuated by 1.9 years.

The findings suggest that the increasing prevalence of chronic diseases with age may help to explain some of the age-related average patterns in personality development, especially in old age. In particular, previous studies have reported declining levels of extraversion, conscientiousness, and openness to experience with age (Lucas \& Donnellan 2011; Wortman et al.2012). These developmental trends are in line with the current findings on disease onset and personality change. On the other hand, emotional stability and related 
traits have been shown to increase (Wortman et al. 2012) or remain stable (Lucas \& Donnellan 2011) in adulthood at least up to age 70, after which levels of negative affectivity may increase again (Baird et al. 2010; Jokela et al. 2013a). Thus, the decreasing emotional stability associated with chronic diseases observed in the present study seem to attenuate the otherwise increasing levels of emotional stability with age.

\section{Plausible mechanisms}

Several mechanisms may underlie the observed associations between chronic diseases and changes in personality. First, the present findings of decreasing emotional stability are in agreement with the extant research showing elevated psychological distress associated with chronic diseases (Sharpe \& Curran 2006; de Ridder et al. 2008). The decreasing levels of emotional stability observed in the present study may be partly caused by people's difficulties in coping with the emotional, behavioral, and cognitive challenges that are posed by the diagnosis of a pernicious condition. Low emotional stability has been associated with less adaptive and less successful coping mechanisms, including avoidance and rumination (Penley et al. 2002; Carver \& Connor-Smith 2010).

Chronic diseases often induce symptoms of fatigue and lethargy, and decrease the person's available energy for physical, emotional, and social activities (de Ridder et al. 2008). This may reflect, at least in part, the infectious and inflammatory processes that cause various non-specific psychosomatic symptoms, such as depressed mood (Raison \& Miller 2012), sleeping problems (Kivimäki et al. 2013), and fatigue (Cho et al. 2012). In terms of personality change, these physiological changes may be expressed as lower levels of extraversion because extraversion entails positive mood, sensitivity to rewarding experiences, and active engagement with the external world. The present results also suggest that decreasing extraversion levels may be particularly linked to the worsening general health 
induced by chronic diseases. Adjusting for change in depressive symptoms and self-rated health explained more than $60 \%$ of the association of chronic disease with change in extraversion, while the corresponding proportion was $36 \%$ or less for the other personality traits.

Of the five personality dimensions studied, conscientiousness is the trait most strongly associated with health behaviors and health outcomes (Bogg \& Roberts 2004; Martin et al. 2007), including premature mortality (Jokela et al. 2013b), obesity (Jokela et al. 2013d), and adult-onset diabetes (Jokela et al. in press-a). The current results suggest that conscientiousness may also be the trait that is most influenced by deteriorating health, as the disease-related change was the largest for conscientiousness. It seems plausible that one of the emotional and cognitive costs of chronic diseases is a decrease in the ability to organize daily activities and fulfill responsibilities in relation to work, family, and personal life, which may become expressed as decreasing levels of conscientiousness. The decrease in openness to experience might also reflect the cognitive costs of chronic morbidity, such as difficulties in the ability to concentrate, willingness to try out new things, and a preference for sticking to existing routines and habits. Except for the brain-related effects of stroke, the associations with openness to experience appear to represent the cumulative effect of morbidity rather than an impact of any specific disease, as the change in openness was observed mainly with the cumulative number of diseases.

As noted in the introduction, personality change due to physiological consequences of a medical condition is one of the diagnostic categories of personality disorders (code F07.0 in ICD-10-CM; code 3.17.11 in DSM-5). The diagnosis mainly considers diseases that directly affect the brain (i.e., clear evidence for physiological mediation) rather than common chronic diseases that may involve both psychosocial adjustment and distal physiological effects (e.g., metabolic and inflammatory responses, e.g., Jokela et al. in press-b). In DSM-5, the five main 
types of such personality change include the labile, disinhibited, aggressive, apathetic, and paranoid types. Conceptually, labile type would correspond to low emotional stability, disinhibited to low conscientiousness, and apathatic to low extraversion. These changes were observed for most of the diseases in the present study, suggesting some commonalities. Aggressive and paranoid types, in turn, would correspond to low agreeableness, but this change was observed only for stroke and respiratory diseases. Specific personality disorders have been associated with more detailed five-factor trait profiles (Saulsman \& Page 2004). The current studies did not include data to separate the contributions of physiological and non-physiological pathways in personality change, and the five-factor traits only partly cover the maladaptive aspects of personality variation (Saulsman \& Page 2004). Additional studies addressing these methodological limitations are needed to assess the clinical relevance of personality changes in response to common chronic diseases.

\section{Associations of specific chronic conditions}

With respect to specific diseases, the largest personality changes were observed for stroke, involving decreasing levels in all five higher-level personality traits. This provides support for the anecdotal evidence of major personality changes often reported in stroke survivors by family members and friends (Stone et al. 2004). Decreasing agreeableness associated with stroke - a change not observed in relation to the other chronic diseases examined in our analysis - might reflect increasing levels of irritability, aggressiveness, and a lack of motivation or energy to reconcile interpersonal conflicts. Similar findings have been reported in relation to degenerative brain diseases, such as dementia and Alzheimer's disease, but with much larger effect sizes of between 10 and $30 \mathrm{~T}$-scores decreases in conscientiousness, emotional stability, and interpersonal warmth (Robins Wahlin \& Byrne 2010). Although one would expect more marked personality change in degenerative brain 
diseases than in stroke, it should also be noted that most of the previous studies have relied on retrospective reports by informants, which may confound the assessment of true personality change with perceived personality change (Franzén-Dahlin et al. 2006).

The second-largest change in personality was observed in relation to respiratory diseases. Chronic obstructive pulmonary disease (COPD) is a particularly disabling condition, greatly limiting a person's physical activities (McCathie et al. 2002). The personality changes associated with respiratory disease and, to a lesser extent, with hypertension, heart problems, and arthritis, indicate that chronic illnesses do not have to affect the brain directly in order to induce systematic personality change. In fact, our findings suggest that the degree of personality change may not be directly related to the degree of physical discomfort or functional impairment caused by the disease. For example, the overall personality change was somewhat larger for hypertension than for arthritis even though hypertension may be symptom-free while arthiritis is characterized by considerable physical pain (Evers et al. 2011). On the other hand, hypertension is one of the strongest predictors of stroke and may be associated with micro-vascular changes in the brain (Go et al. 2013).

Interestingly, cancer was not associated with long-term change in any of the five personality traits. This is surprising, because cancer is clearly a disease with considerable psychological impact (Reich 2008), and adjustment to cancer is often thought to introduce major changes in cancer survivors' cognitive-emotional styles, social behavior, and general outlook on life (Hulbert-Williams et al. 2010). With the focus on long-term personality change, the present study included only long-term cancer survivors. While the short-term psychological impact of cancer is likely to be particularly severe (Reich 2008), it is possible that cancer survivors adapt to their life circumstances over time. Cancer is also the only disease included in the present analysis that is not necessarily a progressive condition but from which a full recovery is possible. It is possible that the adverse psychological impact of 
cancer is counterbalanced by the relief of overcoming the disease, and that there are no longterm changes in basic personality dispositions among individuals who have completely or partially recovered from cancer.

\section{Strengths and limitations}

The notable strengths of the present study include prospective data from four cohort studies, and the use of standardized personality measures. The lack of multiple repeated measurements of personality precluded a more detailed analysis of temporal trajectories, that is, how different aspects of personality are affected immediately after the first symptoms of a chronic disease, and how these changes attenuate or exacerbate over time. However, it is worth noting that comparisons of cohort-specific associations did not suggest that the extent of personality change was associated with the length of follow-up (Supplementary Figures S2-S13). Another methodological limitation was the reliance on self-reported data on chronic diseases, which underestimates the true prevalence of diseases. Third, because observational study design cannot establish causal associations with certainty, some of the associations might have been confounded by unobserved variables related to aging process or disease onset. This appears unlikely as (1) not all personality traits changed in the same average direction associated with aging (i.e., emotional stability decreased with the onset of diseases whereas the average age-related change was increasing emotional stability), and (2) the unobserved confounder would need to explain why different chronic diseases are associated with different degrees of personality change.

It is possible that the assessment of mean changes in personality associated with disease onset conceals important individual differences in the nature and magnitude of changes in personality. It has been suggested that some individuals might be able to use their adverse experiences with a disease to facilitate personal growth and greater appreciation of positive 
aspects of their lives (Barskova \& Oesterreich 2009; Bostock et al.2009). Thus, individual variation and potential beneficial personality changes in other psychological dispositions besides basic personality dimensions associated with disease onset require further investigation.

\section{Conclusion}

Data from over 17,000 individuals suggest that the onset of chronic diseases is associated with changes in four of the five major dimensions of personality assessed by the five-factor model. For extraversion, conscientiousness, and openness to experience, the age-related average decline in these personality traits in the population is accelerated by approximately 1.5 to 5.5 years of age for each disease, while the age-related increase in emotional stability was set back by about 2 years. This individual-participant meta-analysis provides strong evidence to suggest that common chronic diseases modify personality development throughout the adult life span. 
Table 1. Descriptive statistics of the samples

\begin{tabular}{|c|c|c|c|c|}
\hline & HRS & MIDUS & WLSG & WLSS \\
\hline \multicolumn{5}{|l|}{ Basic characteristics } \\
\hline Sex (\% females) & $58.6(3,013)$ & $55.5(2,130)$ & $54.9(3,027)$ & $54.9(1,647)$ \\
\hline Racial/Ethnic minority (\%) & $18.5(952)$ & $7.1(273)$ & - & - \\
\hline Age at T1 (years) & $66.0(9.8)$ & $47.2(12.4)$ & $54.1(0.5)$ & $52.7(7.0)$ \\
\hline Age range at $\mathrm{T} 1$ (min-max) & $25-104$ & $20-75$ & $53-56$ & $33-95$ \\
\hline Follow-up time T1-T2 (years) & $4.2(0.4)$ & $8.9(0.5)$ & $11.2(0.3)$ & $11.3(0.6)$ \\
\hline \multicolumn{5}{|l|}{ Outcome variables* } \\
\hline Extraversion T2 & $49.1(10.5)$ & $48.3(10.3)$ & $49.5(9.8)$ & $49.7(9.2)$ \\
\hline Emotional Stability T2 & $51.3(10.2)$ & $52.0(9.4)$ & $51.8(9.3)$ & $51.5(9.1)$ \\
\hline Agreeableness T2 & $49.7(10.6)$ & $49.3(10.2)$ & $50.5(9.7)$ & $50.3(9.3)$ \\
\hline Conscientiousness T2 & $49.3(11.0)$ & $50.8(10.3)$ & $48.9(10.2)$ & $49.1(9.5)$ \\
\hline Openness to Experience T2 & $48.7(10.5)$ & $47.9(10.4)$ & $49.2(9.6)$ & $49.4(9.4)$ \\
\hline Self-rated health T2 & $48.9(9.9)$ & $49.3(10.8)$ & $43.8(14.9)$ & $42.8(14.9)$ \\
\hline Depressive symptoms T2 & $50.0(9.9)$ & $49.4(9.3)$ & $48.8(9.6)$ & $49.2(9.6)$ \\
\hline \multicolumn{5}{|l|}{ Chronic diseases } \\
\hline Heart disease T1 (\%) & $21.4(1,100)$ & $11.4(439)$ & $5.0(273)$ & $7.8(229)$ \\
\hline Heart disease T2 (\%) & $28.5(1,465)$ & $17.8(682)$ & $14.6(773)$ & $14.6(408)$ \\
\hline Hypertension T1 (\%) & $55.5(2,854)$ & $16.3(622)$ & $20.5(1,128)$ & $24.9(735)$ \\
\hline Hypertension T2 (\%) & $63.9(3,287)$ & $30.3(1,162)$ & $47.3(2,504)$ & $46.8(1,307)$ \\
\hline Cancer T1 (\%) & $13.8(711)$ & $7.3(279)$ & $1.8(101)$ & $6.4(188)$ \\
\hline Cancer T2 (\%) & $18.5(950)$ & $14.1(541)$ & $10.7(565)$ & $10.8(302)$ \\
\hline Diabetes T1 (\%) & $18.2(934)$ & $3.9(149)$ & $3.4(185)$ & $3.9(116)$ \\
\hline Diabetes T2 (\%) & $24.0(1,235)$ & $10.1(387)$ & $11.8(624)$ & $11.5(322)$ \\
\hline Respiratory disease T1 (\%) & $9.3(479)$ & $12.9(490)$ & $6.7(372)$ & $17.4(510)$ \\
\hline Respiratory disease T2 (\%) & $12.4(635)$ & $12.7(489)$ & $12.7(686)$ & $12.8(373)$ \\
\hline Arthritis T1 (\%) & $60.2(3,094)$ & $19.6(747)$ & $23.0(1,266)$ & $31.0(914)$ \\
\hline Arthritis T2 (\%) & $68.2(3,505)$ & $26.2(1,007)$ & $45.1(2,389)$ & $45.0(1,256)$ \\
\hline Stroke T1 (\%) & $4.3(221)$ & $0.4(14)$ & $0.6(32)$ & $0.9(24)$ \\
\hline Stroke T2 (\%) & $6.8(352)$ & $1.0(40)$ & $2.8(149)$ & $3.5(99)$ \\
\hline Total number of participants & $100.0(5,140)$ & $100.0(3,838)$ & $100.0(5,515)$ & $100.0(3,000)$ \\
\hline
\end{tabular}

Note: For continuous variables, values are means (and standard deviations). * Outcome variables are standardized as $T$-scores against baseline values, so $T 1$ mean $=50$ and $S D=10$ for all outcome variables. For categorical variables, values are percentages (and number of participants). 
Table 2. Pooled associations between chronic diseases and personality change, adjusted for concurrent change in depressive symptoms and self-rated health.

\section{Personality trait}

\begin{tabular}{|c|c|c|c|c|c|}
\hline Adjusted for: & $E$ & $S$ & $A$ & C & 0 \\
\hline \multirow{2}{*}{ Sex, age, race } & -0.25 & -0.40 & -0.05 & -0.44 & -0.25 \\
\hline & $(-0.40,-0.10)$ & $(-0.61,-0.19)$ & $(-0.19,0.08)$ & $(-0.57,-0.30)$ & $(-0.37,-0.13)$ \\
\hline + Depressive & -0.23 & -0.36 & -0.04 & -0.41 & -0.24 \\
\hline symptoms & $(-0.39,-0.07)$ & $(-0.58,-0.14)$ & $(-0.18,0.09)$ & $(-0.55,-0.27)$ & $(-0.37,-0.12)$ \\
\hline \multirow{2}{*}{ + Self-rated health } & -0.11 & -0.28 & 0.03 & -0.30 & -0.16 \\
\hline & $(-0.26,0.05)$ & $(-0.54,-0.02)$ & $(-0.11,0.16)$ & $(-0.44,-0.16)$ & $(-0.29,-0.04)$ \\
\hline \multirow{2}{*}{+ All above } & -0.10 & -0.26 & 0.03 & -0.29 & -0.16 \\
\hline & $(-0.25,0.06)$ & $(-0.53,0.01)$ & $(-0.11,0.17)$ & $(-0.43,-0.14)$ & $(-0.29,-0.03)$ \\
\hline
\end{tabular}

$\begin{array}{llllll}\text { Total attenuation (\%) } & 61.8 & 35.7 & - & 34.4 & 36.8\end{array}$

Note: Values are regression coefficients (and 95\% confidence intervals) indicating linear change in personality trait T-score per 1 chronic disease between baseline and follow-up. $N=16,454$ due to

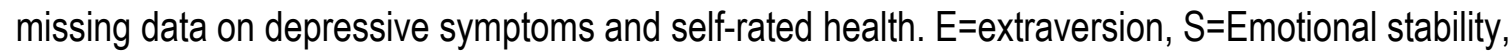
$\mathrm{A}=$ Agreeableness, $\mathrm{C}=$ Conscientiousness, $\mathrm{O}=$ Openness to Experience. 
Figure legends

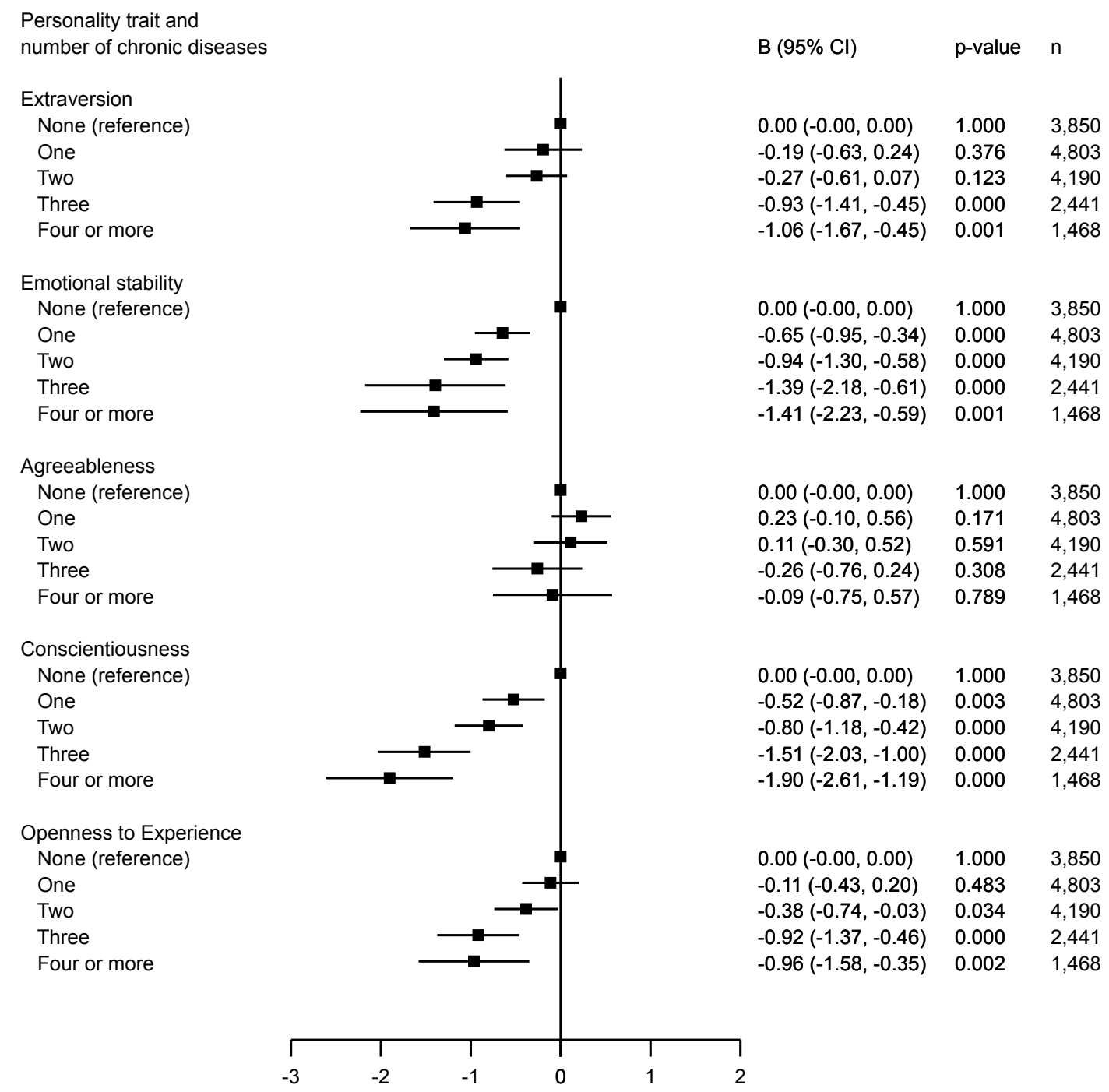

Figure 1. Associations between the number of onset chronic disease and personality change between baseline and follow-up phase in the pooled sample (random-effect meta-analysis), adjusted for sex, age, race/ethnicity, follow-up time, and number of chronic diseases at baseline. See Online Supplementary Figures S2-S6 for cohort-specific details. 


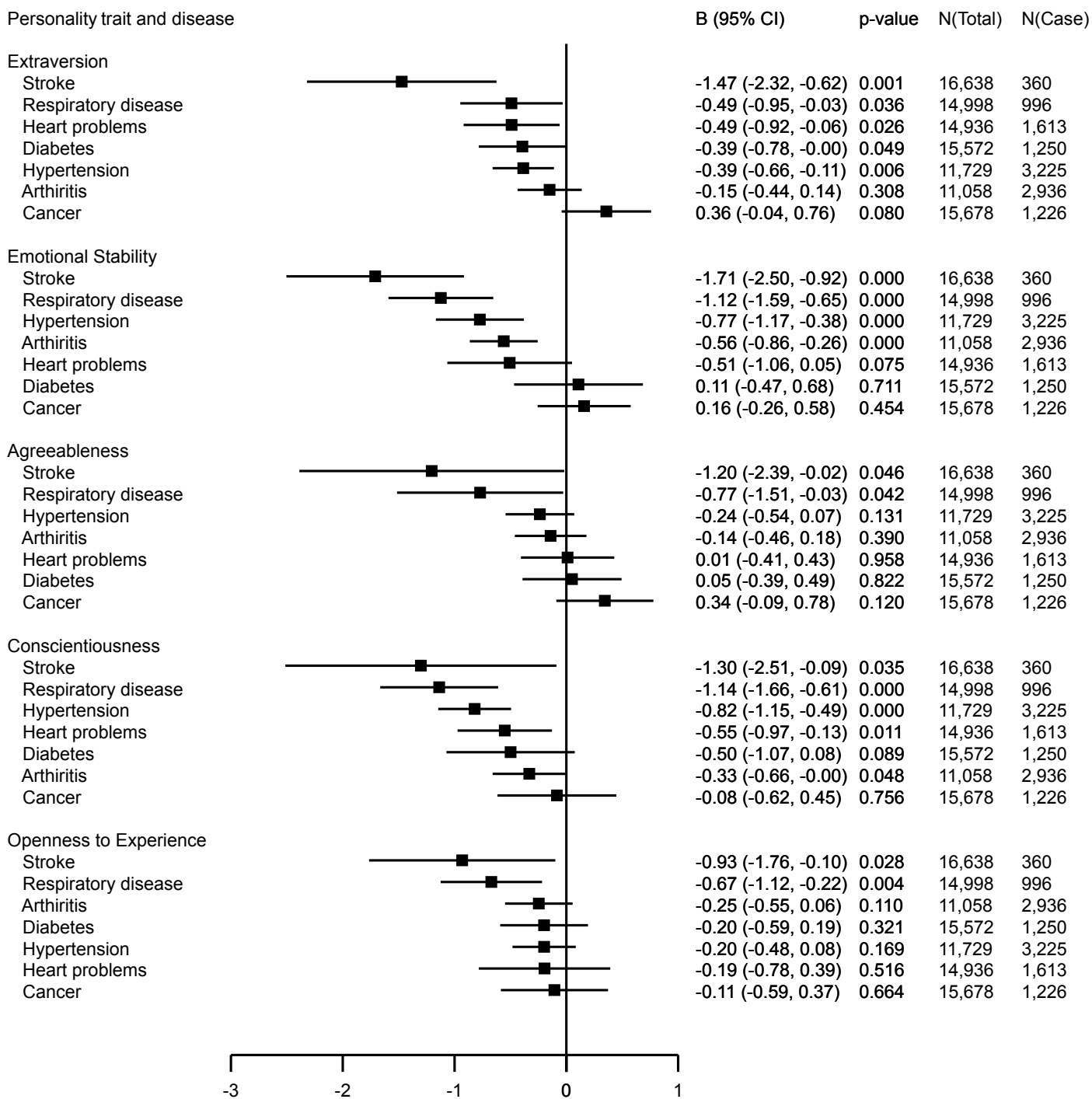

Figure 2. Personality change associated with the onset of different chronic diseases in participants free of the chronic disease of interest at baseline, adjusted for sex, age, race/ethnicity, follow-up time, and the number of chronic diseases at baseline. A separate meta-analysis was carried out for each chronic disease and personality trait, and the pooled estimates of randomeffect meta-analysis are plotted in the figure (see Online Supplementary Figures S7-S13 for cohort-specific details).

Estimates within each personality trait are sorted in order of effect magnitude of the disease. 


\section{Acknowledgements}

The authors wish to gratefully thank the original collectors of the data, and the ESDS (Economic and Social Data Service; http://www.esds.ac.uk/) and ICPSR (Inter-university Consortium for Political and Social Research; www.icpsr.umich.edu) for making the data available. The research uses data from the Wisconsin Longitudinal Study (WLS) of the University of Wisconsin-Madison. Since 1991, the WLS has been supported principally by the National Institute on Aging (AG-9775 and AG-21079), with additional support from the Vilas Estate Trust, the National Science Foundation, the Spencer Foundation, and the Graduate School of the University of Wisconsin-Madison. A public use file of data from the Wisconsin Longitudinal Study is available from the Wisconsin Longitudinal Study, University of Wisconsin-Madison, 1180 Observatory Drive, Madison, Wisconsin 53706 and at http://www.ssc.wisc.edu/wlsresearch/data/. The interpretations, opinions, and inferences based on the data are solely the responsibility of the authors.

\section{References}

Affleck G, Tennen H, Zautra A, \& Urrows S (2001). Women's pursuit of personal goals in daily life with fibromyalgia: A value-expectancy analysis. Journal of Consulting and Clinical Psychology 69, 587-596.

Baird BM, Lucas RE, \& Donnellan MB (2010). Life satisfaction across the lifespan: Findings from two nationally representative panel studies. Social Indicators Research 99, 183-203.

Barskova T, \& Oesterreich R (2009). Post-traumatic growth in people living with a serious medical condition and its relations to physical and mental health: a systematic review. 
Disability and Rehabilitation 31, 1709-1733.

Bogg T, \& Roberts BW (2004). Conscientiousness and health-related behaviors: A metaanalysis of the leading behavioral contributors to mortality. Psychological Bulletin 130, 887919.

Bostock L, Sheikh AI, \& Barton S (2009). Posttraumatic growth and optimism in healthrelated trauma: A systematic review. Journal of Clinical Psychology in Medical Settings 16, 281-296.

Brim OG, Baltes PB, \& Bumpass LL (2007). National Survey of Midlife Development in the United States (MIDUS), 1995-1996. [computer file]. ICPSR02760-v4. Ann Arbor, MI: DataStat, Inc./Boston, MA: Harvard Medical School, Dept. of Health Care Policy [producers]. Ann Arbor, MI: Inter-university Consortium for Political and Social Research [distributor], 2007-04-16; 2007

Carver CS, \& Connor-Smith J (2010). Personality and coping. Annual Review of Psychology 61, 679-704.

Cho HJ, Kivimäki M, Bower JE, \& Irwin MR (2012). Association of C-reactive protein and interleukin-6 with new-onset fatigue in the Whitehall II prospective cohort study. Psychological Medicine 43, 1-11.

Clark AE, Diener E, Georgellis Y, \& Lucas RE (2008). Lags and leads in life satisfaction: A test of the baseline hypothesis. The Economic Journal 118, F222-F243.

Crimmins EM (2004). Trends in the health of the elderly. Annual Review of Public Health 25, 79-98.

Davydov DM, Stewart R, Ritchie K, \& Chaudieu I (2010). Resilience and mental health. 
Clinical Psychology Review 30, 479-495.

de Ridder D, Geenen R, Kuijer R, \& van Middendorp H (2008). Psychological adjustment to chronic disease. Lancet 372, 246-255.

Evers AWM, Zautra A, \& Thieme K (2011). Stress and resilience in rheumatic diseases: a review and glimpse into the future. Nature Reviews Rheumatology 7, 409-415.

Franzén-Dahlin Å, Billing E, Näsman P, Mårtensson B, Wredling R, \& Murray V (2006). Post-stroke depression - effect on the life situation of the significant other. Scandinavian Journal of Caring Sciences 20, 412-416.

Gao S, Dolan N, Hall KS, \& Hendrie HC (2000). The association of demographic factors and physical illness with personality change in a community sample of elderly African Americans. The American Journal of Geriatric Psychiatry 8, 209-214.

Go AS, Mozaffarian D, Roger VL, Benjamin EJ, Berry JD, Borden WB, Bravata DM, Dai S, Ford ES, Fox CS, Franco S, Fullerton HJ, Gillespie C, Hailpern SM, Heit JA, Howard VJ, Huffman MD, Kissela BM, Kittner SJ, Lackland DT, Lichtman JH, Lisabeth LD, Magid D, Marcus GM, Marelli A, Matchar DB, McGuire DK, Mohler ER, Moy CS, Mussolino ME, Nichol G, Paynter NP, Schreiner PJ, Sorlie PD, Stein J, Turan TN, Virani SS, Wong ND, Woo D, Turner MB, American Heart Association Statistics Committee and Stroke Statistics Subcommittee (2013). Executive summary: Heart disease and stroke statistics - 2013 update: a report from the American Heart Association.

Circulation 127, 143-152.

Headey B (2010). The set point theory of well-being has serious flaws: On the eve of a scientific revolution? Social Indicators Research 97, 7-21. 
Hulbert-Williams N, Neal R, Morrison V, Hood K, \& Wilkinson C (2011). Anxiety, depression and quality of life after cancer diagnosis: what psychosocial variables best predict how patients adjust? Psycho-Oncology 21, 857-867.

Jokela M, Batty GD, \& Kivimäki M (2013a). Ageing and the prevalence and treatment of mental health problems. Psychological Medicine 43, 2037-2045.

Jokela M, Batty GD, Nyberg ST, Virtanen M, Nabi H, Singh-Manoux A, \& Kivimäki M (2013b). Personality and all-cause mortality: Individual-participant meta-analysis of 3,947 deaths in 76,150 adults. American journal of epidemiology 178, 667-675.

Jokela M, Elovainio M, Nyberg ST, Tabák AG, Hintsa T, Batty GD, \& Kivimäki M (in press-a). Personality and risk of diabetes in adults: Pooled analysis of 5 cohort studies. Health Psychology, in press. doi:10.1037/hea0000003

Jokela M, Hamer M, Singh-Manoux A, Batty GD, Kivimäki M. (in press-b). Association of metabolically healthy obesity with depressive symptoms: pooled analysis of eight studies. Molecular Psychiatry. doi:10.1038/mp.2013.162

Jokela M, Hintsanen M, Hakulinen C, Batty GD, Nabi H, Singh-Manoux A, \& Kivimäki M (2013d). Association of personality with the development and persistence of obesity: a meta-analysis based on individual-participant data. Obesity Reviews 14, 315-323.

Juster FT, \& Suzman R (1995). An overview of the Health and Retirement Study. Journal of Human Resources 30, S7-S56.

Katz PP, \& Yelin EH (1995). The development of depressive symptoms among women with rheumatoid arthritis. The role of function. Arthritis and Rheumatism 38, 49-56.

Kivimäki M, Shipley MJ, Batty GD, Hamer M, Akbaraly TN, Kumari M, Jokela M, 
Virtanen M, Lowe GD, Ebmeier KP, Brunner EJ, \& Singh-Manoux A (in press). Longterm inflammation increases risk of common mental disorder: a cohort study. Molecular Psychiatry. doi:10.1038/mp.2013.35

Lucas RE, \& Donnellan MB (2011). Personality development across the life span: Longitudinal analyses with a national sample from Germany. Journal of Personality and Social Psychology 101, 847-861.

Martin LR, Friedman HS, \& Schwartz JE (2007). Personality and mortality risk across the life span: The importance of conscientiousness as a biopsychosocial attribute. Health Psychology 26, 428-436.

McCathie HCF, Spence SH, \& Tate RL (2002). Adjustment to chronic obstructive pulmonary disease: the importance of psychological factors. European Respiratory Journal 19, 47-53.

Mendelsohn GA, Dakof GA, \& Skaff M (1995). Personality change in Parkinson's disease patients: Chronic disease and aging. Journal of Personality 63, 233-257.

Nelson LD, Cicchetti D, Satz P, Stern S, Sowa M, Cohen S, Mitrushina M, \& van Gorp W (1993). Emotional sequelae of stroke. Neuropsychology 7, 553-560.

Obonsawin MC, Jefferis S, Lowe R, Crawford JR, Fernandes J, Holland L, Woldt K, Worthington E, \& Bowie G (2007). A model of personality change after traumatic brain injury and the development of the brain injury personality scales. Journal of Neurology, Neurosurgery \& Psychiatry 78, 1239-1247.

Osterberg L, \& Blaschke T (2005). Adherence to medication, New England Journal of Medicine 353, 487-497. 
Penley JA, Tomaka J, \& Wiebe JS (2002). The association of coping to physical and psychological health outcomes: A meta-analytic review. Journal of Behavioral Medicine 25, $551-603$.

Powdthavee N (2009). What happens to people before and after disability? Focusing effects, lead effects, and adaptation in different areas of life. Social Science \& Medicine 69, 18341844.

Prince M, Patel V, Saxena S, Maj M, Maselko J, Phillips MR, \& Rahman A (2007). No health without mental health. Lancet 370, 859-877.

Raison CL, \& Miller AH (2013). The evolutionary significance of depression in Pathogen Host Defense (PATHOS-D). Molecular Psychiatry 18, 15-37.

Reich M (2008). Depression and cancer: recent data on clinical issues, research challenges and treatment approaches. Current Opinion in Oncology 20, 353-359.

Remer-Osborn J (1998). Psychological, behavioral, and environmental influences on poststroke recovery. Topics in Stroke Rehabilitation 5, 45-53.

Robins Wahlin T-B, \& Byrne GJ (2010). Personality changes in Alzheimer's disease: a systematic review. International Journal of Geriatric Psychiatry 26, 1019-1029.

Saulsman LM, \& Page AC (2004). The five-factor model and personality disorder empirical literature: A meta-analytic review. Clinical Psychology Review 23, 1055-1085.

Sharpe L, \& Curran L (2006). Understanding the process of adjustment to illness. Social Science \& Medicine 62, 1153-1166.

Sheikh AI (2004). Posttraumatic growth in the context of heart disease. Journal of Clinical 
Psychology in Medical Settings 11, 265-273.

Stanton AL, Revenson TA, \& Tennen H (2007). Psychological adjustment to chronic disease. Annual Review of Psychology 58, 565-592.

Stone J, Townend E, Kwan J, Haga K, Dennis MS, \& Sharpe M (2004). Personality change after stroke: Some preliminary observations. British Medical Journal 75, 1708-1713. Strong K, Mathers C, Leeder S, \& Beaglehole R (2005). Preventing chronic diseases: how many lives can we save? Lancet 366, 1578-1582.

\section{Wang H, Dwyer-Lindgren L, Lofgren KT, Rajaratnam JK, Marcus JR, Levin-Rector}

A, Levitz CE, Lopez AD, \& Murray PCJ (2012). Age-specific and sex-specific mortality in 187 countries, 1970-2010: a systematic analysis for the Global Burden of Disease Study 2010. Lancet 380, 2071-2094.

Wollmering (2007). Wisconsin Longitudinal Study Handbook (12.10.07). 2007.

Wortman J, Lucas RE, \& Donnellan MB (2012). Stability and change in the Big Five personality domains: Evidence from a longitudinal study of Australians. Psychology and Aging 27, 867-874.

Wu S (2001). Adapting to heart conditions: A test of the hedonic treadmill. Journal of Health Economics 20, 495-508. 